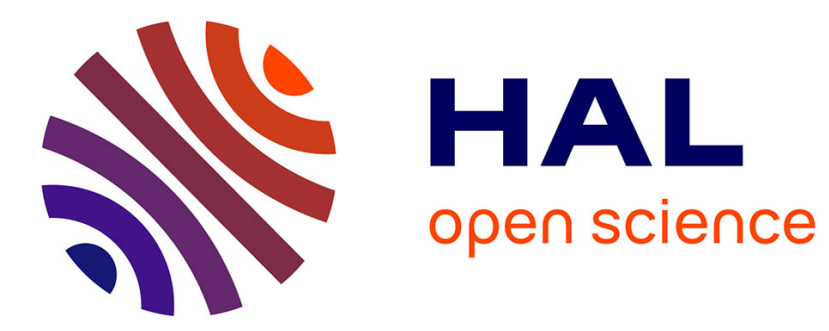

\title{
PERCEPTUAL CURVE EXTRACTION
}

\author{
Baptiste Magnier, Daniel Diep, Philippe Montesinos
}

\section{To cite this version:}

Baptiste Magnier, Daniel Diep, Philippe Montesinos. PERCEPTUAL CURVE EXTRACTION. IEEE IVMSP (Image, Video, and Multidimensional Signal Processing Technical Committee) on "Perception and Visual Signal Analysis", Jun 2011, Ithaca, United States. http://www.ivmsp2011.org/. hal00807815

\section{HAL Id: hal-00807815 https://hal.science/hal-00807815}

Submitted on 4 Apr 2013

HAL is a multi-disciplinary open access archive for the deposit and dissemination of scientific research documents, whether they are published or not. The documents may come from teaching and research institutions in France or abroad, or from public or private research centers.
L'archive ouverte pluridisciplinaire HAL, est destinée au dépôt et à la diffusion de documents scientifiques de niveau recherche, publiés ou non, émanant des établissements d'enseignement et de recherche français ou étrangers, des laboratoires publics ou privés. 


\title{
PERCEPTUAL CURVE EXTRACTION
}

\author{
Baptiste Magnier, Daniel Diep, Philippe Montesinos.
}

\author{
Ecole des Mines d'ALES, LGI2P, \\ Site EERIE, Parc Scientifique G.Besse \\ 30035 Nimes Cedex 1 \\ \{Baptiste.Magnier, Daniel.Diep, Philippe.Montesinos\}@mines-ales.fr
}

\begin{abstract}
In this paper we propose a new perceptual curve detection method in images based on the difference of half rotating Gaussian filters. The novelty of this approach resides in the mixing of ideas coming both from directional filters, perceptual organization and DoG method. We obtain a new anisotropic DoG detector enabling very precise detection of perceptual curve points. Moreover, this detector performs correctly at perceptual curves even if highly bended, and is precise on perceptual junctions. This detector has been tested successfully on various image types presenting real difficult problems for classical detection methods.
\end{abstract}

Index Terms - Perceptual grouping, curve extraction, ridge/valley detection, anisotropic DoG filters.

\section{INTRODUCTION}

In computer vision, perceptual organization has been often used for the extraction of curves in images. Many works address the problem of qualifying visual quality of curves which is often referenced as saliency [1] [2] [3] [4] [5]. In this paper we revisit perceptual grouping and saliency with an anisotropic filtering point of view. At the contrary of most of the works concerning perceptual organization, the method proposed here is not only able to deal with binary line images but is also able to segment directly complex images such as aerial images or enhance gradient images, for example, for edge detection purpose.

Anisotropic filters take an important part in image processing. Indeed, anisotropic filters provide good results and are often used in edge detection [6] [7], texture removal [8], image enhancing and restoration [9]. In several domains, anisotropic filters make possible better robustness than classical method. However, they are seldom used in line or curve detection.

A curve in an image can be represented by a ridge or a valley on the image surface, ie. points where the gray level reaches a local extremum in a given direction (illustrated in Fig. 1). This direction is the normal to the curve lying along

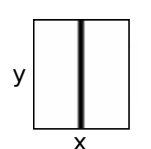

(a)

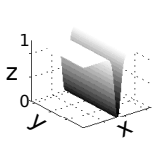

(b)

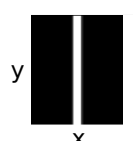

(c)

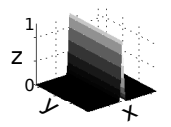

(d)

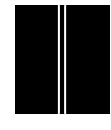

(e)
Fig. 1. Valley and ridge in scalar images. (a) Valley in an image. (b) Surface representation of a valley. (c) Ridge in an image. (d) Surface representation of a ridge. The z axis corresponds to the intensity gray level. (e) Edge detection on the image in (a).

the ridge (or respectively the valley). Ridges and valleys correspond to important features in images. For example in aerial images, they are often attached to roads [10], they may also be issued from edge detection method, or they can simply belong to curves in line drawing images.

Classical edge detection [11] fails to detect ridges or valleys in images as it provides two edges at each side of the ridge or the valley (illustrated in Fig. 1(e)). On dashed lines images, applying edge detection methods result in small squares and applying anisotropic edge detection methods [6] [7] result in two parallel lines (illustrated in Fig. 2).

Lines extraction methods can be divided in three main categories of segmentation algorithms. The first refers to pattern recognition and filtering techniques (for examples differential geometry [10] and morphology), the second to model-based approaches (snakes) [12], and the third to tracking-based approaches.

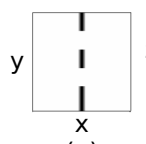

(a)

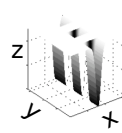

(b)

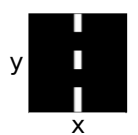

(c)

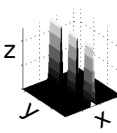

(d)

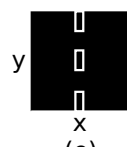

(e)

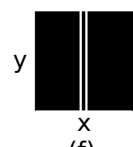

(f)
Fig. 2. Discontinuous valley and ridge in scalar images. (a) Discontinuous valley in an image. (b) Surface representation of (a). (c) Ridge in an image. (d) Surface representation of a ridge. The $z$ axis corresponds to the intensity gray level. (e) Edge detection on the image in (a). (f) Anisotropic edge detection on the image in (a) 
Filtering techniques [10] are well adapted in ridge and valley extraction because they are able to smooth the noise and amplify the curve information by computing surface curvature. However, results obtained by these approaches can present important false detection rate in noisy images. It is mainly because the high pass the second derivative filtering is sensitive to noise.

In this paper, we present a rotating filter (inspired by [7], [8] and [10]) able to detect ridges and valleys. Our curve detector involves anisotropic directional linear filtering by means of difference of two half rotating smoothing filters. Then, we compute a ridge or valley operator using a local directional maximization or respectively minimization of the response of the filters. Contrary to several approaches involving curves, the method presented here performs fine even on highly bended ridges or valleys. Finally, due to its strong smoothing in the directions (we define two directions for a curve crossing a pixel : entering and leaving directions) of the curve, the detection is not sensitive to noise. Furthermore, as the method proposed here involves recursive filters, the computational time is small, then it could be easily implemented for real-time video.

This paper is organized as follows. In the section 2, we present an anisotropic smoothing half Gaussian filter. We present a robust curve detector using difference of two half directional Gaussian filters in the section 3. The section 4 is devoted to experimental results. Finally, the section 5 concludes this paper.

\section{A ROTATING SMOOTHING FILTER}

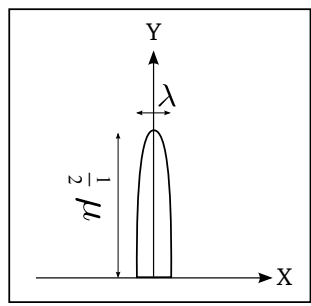

(a) Smoothing filter

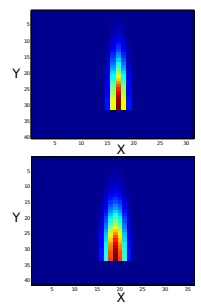

(b) Discretized filter

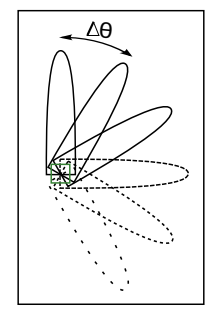

(c) Rotating filters
Fig. 3. A smoothing rotating filter. For (b) top: $\mu=10$ and $\lambda=1$. For (b) bottom: $\mu=10$ and $\lambda=1.5$.

In our method, for each pixel of the original image, we use a rotating smoothing filter in order to build a signal $s$ which is a function of a rotation angle $\theta$ and the underlying signal. As shown in [7] and [8], smoothing with rotating filters means that the image is smoothed with a bank of rotated anisotropic Gaussian kernels:

$$
G_{(\mu, \lambda)}(x, y, \theta)=C \cdot H\left(R_{\theta}\left(\begin{array}{l}
x \\
y
\end{array}\right)\right) e^{-\left(P_{s}(x, y, \theta)\right)}
$$

with

$$
P_{s}(x, y, \theta)=-\left(\begin{array}{ll}
x & y
\end{array}\right) R_{\theta}^{-1}\left(\begin{array}{ll}
\frac{1}{2 \mu^{2}} & 0 \\
0 & \frac{1}{2 \lambda^{2}}
\end{array}\right) R_{\theta}\left(\begin{array}{l}
x \\
y
\end{array}\right)
$$

where $C$ is a normalization coefficient, $R_{\theta}$ a rotation matrix of angle $\theta, x$ and $y$ are pixel coordinates and $\mu$ and $\lambda$ the standard-deviations of the Gaussian filter (see Fig. 3(a)).

As we need only the causal part of the filter (illustrated on Fig. 3(a) and (b)), we simply "cut" the smoothing kernel by the middle, this operation corresponds to the Heaviside function $H$ [7]. By convolution with these rotated kernels (see figure 3(b)), we obtain a collection of directional smoothed images $I_{\theta}=I * G_{(\mu, \lambda)}(\theta)$.

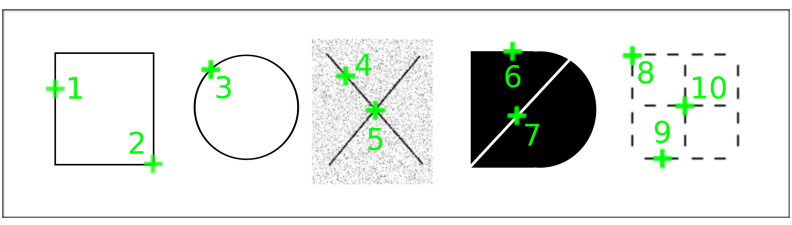

Fig. 4. Points selection on an original image.

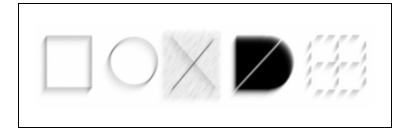

(a) $\theta=34$ degrees, $\mu=10, \lambda=1$

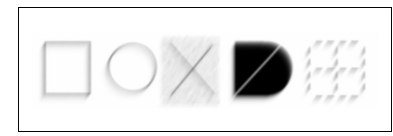

(c) $\theta=34$ degrees, $\mu=10, \lambda=1.5$

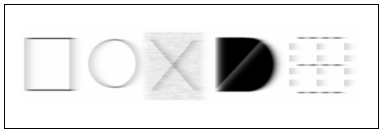

(b) $\theta=270$ degrees, $\mu=10, \lambda=1$

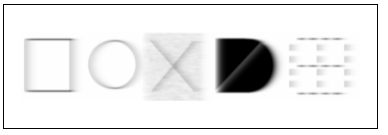

(d) $\theta=270$ degrees, $\mu=10, \lambda=1.5$
Fig. 5. Image in Fig. 4(c) smoothed using different parameters and different orientations.

For computational efficiency, we proceed in a first step to the rotation of the image at some discretized orientations from 0 to 360 degrees (of $\Delta \theta=1,2,5$, or 10 degrees, depending on the angular precision needed and the smoothing parameters) before applying non rotated smoothing filters. $\mu$ and $\lambda$ define the standard-deviations of the Gaussian filter. As the image is rotated instead of the filters, the filtering implementation can use efficient recursive approximation of the Gaussian filter [13]. As presented in [7], the implementation is quite straightforward. In a second step, we apply an inverse rotation of the smoothed image and obtain a bank of $360 / \Delta \theta$ images (some examples are available in Fig. 5). 


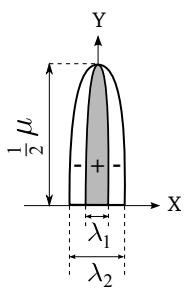

(a) A DRF

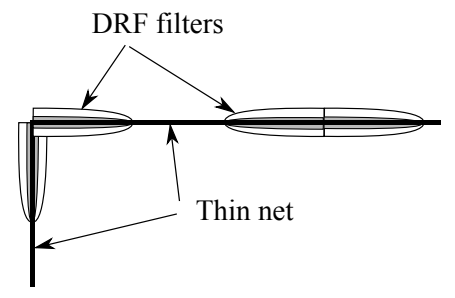

(b) DRF in the thin net directions
Fig. 6. DRF filter descriptions.

\section{PERCEPTUAL RIDGE/VALLEY LINES DETECTION USING DIFFERENCE OF DIRECTIONAL GAUSSIAN FILTERS}

\subsection{Difference of Rotated Half Smoothing Filters (DRF)}

As presented in Fig. 6(a), we want to estimate at each pixel a smoothed second derivative of the image along a curve crossing this pixel. In one dimension, the second derivative of a signal can be estimated thanks to a DoG operator. For our problem, we have just to apply two filters with two different $\lambda$ and the same $\mu$ to obtain directional derivatives. Then, we compute the difference of these two filters to obtain the desired smoothed second derivative information in the curve directions (illustrated in Fig. 6(b)).

\subsection{Pixel Classification}

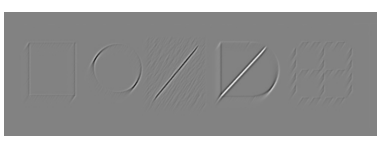

(a) $\theta=34$ degrees

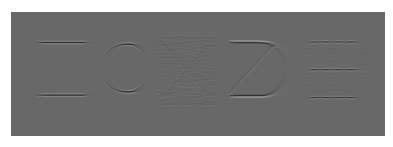

(b) $\theta=270$ degrees
Fig. 7. DRF result of Fig. 4(c) at different orientation $\theta$ using the following parameters: $\mu=10, \lambda_{1}=1$ and $\lambda_{2}=1.5$ (normalized images).

Applying by convolution the DRF filter to each pixel of an image (by means of a technique of rotated images, as defined above), we obtain for each pixel a signal which corresponds to a scan in $360 / \Delta \theta$ directions (see Fig. 7). Our idea is then to characterize pixels which belong to a curve (a ridge or a valley), and thus to build our detector.

Let $D(x, y, \theta)$ be the pixel signal obtained at pixel $\mathrm{P}$ located at $(x, y) . D(x, y, \theta)$ is a function of the direction $\theta$ such that:

$$
D(x, y, \theta)=G_{\left(\mu, \lambda_{1}\right)}(x, y, \theta)-G_{\left(\mu, \lambda_{2}\right)}(x, y, \theta)
$$

where $x$ and $y$ are pixel coordinates. $\mu, \lambda_{1}$ and $\lambda_{2}$ correspond to the standard-deviations of the Gaussians. Some examples are represented on Fig. 8.
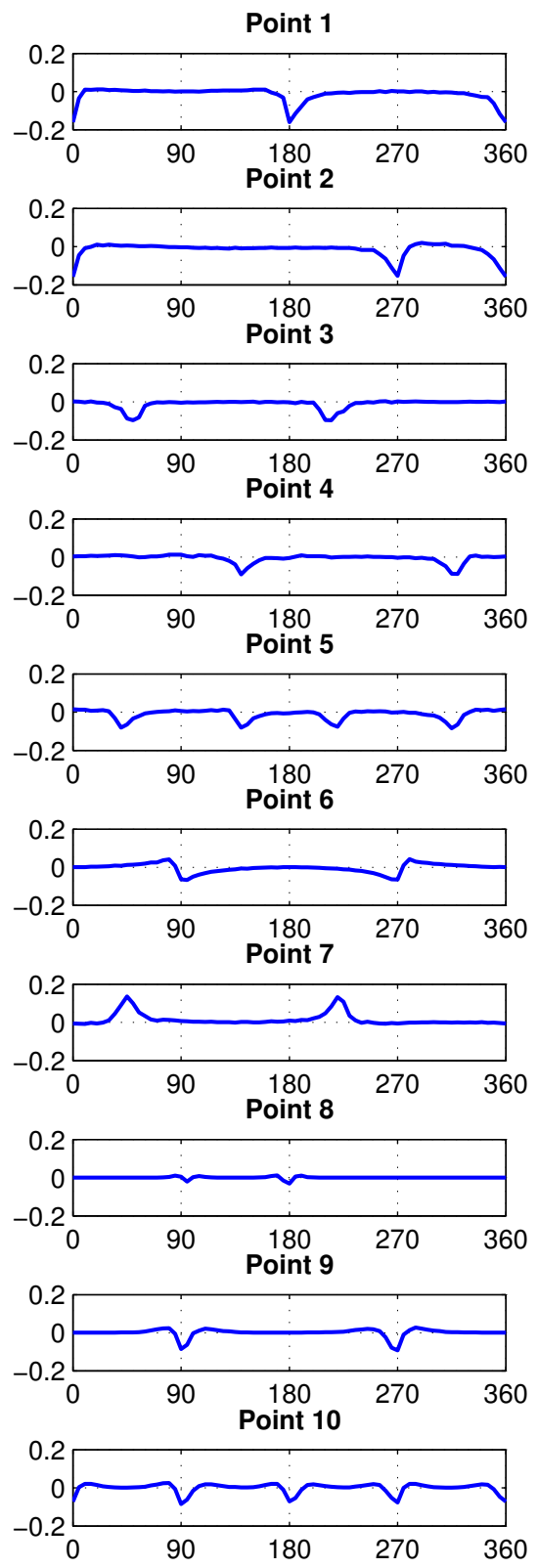

Fig. 8. Examples of functions $D(x, y, \theta)$ on the points selection in Fig. 4(c) using $\mu=10, \lambda_{1}=1, \lambda_{2}=1.5$.. The $x$-axis corresponds to the value of $\theta$ (in degrees) and the $y$-axis to $D(x, y, \theta)$.

We define a ridge/valley operator $\Sigma(x, y)$ by the following expression:

$$
\begin{aligned}
\Sigma(x, y) & =D\left(x, y, \theta_{M_{1}}\right)+D\left(x, y, \theta_{M_{2}}\right) \\
& +D\left(x, y, \theta_{m_{1}}\right)+D\left(x, y, \theta_{m_{2}}\right)
\end{aligned}
$$


where $\theta_{M_{1}}, \theta_{M_{2}}$ are the directions of the local maxima of the function $D$ and $\theta_{m_{1}}, \theta_{m_{2}}$ the directions of the local minima (see example in Fig. 10(a)). Conditions of detection are as follows:

if $\Sigma(x, y)>\Sigma_{t h}$, the pixel $\mathrm{P}$ belongs to a ridge,

if $\Sigma(x, y)<-\Sigma_{t h}$, the pixel P belongs to a valley line, where $\Sigma_{t h}>0$.

On a typical valley (for example point 1, 3 and 4 in Fig. 8), the pixel signal at the minimum of a valley contains at least two negative sharp peaks. For ridges (for example point 7 in Fig. 8), the pixel signal at the maximum of a ridge contains at least two positive peaks. These sharp peaks correspond to the two directions of the curve (an entering and leaving path). In case of a junction, the number of peaks corresponds to the number of curves in the junction (see point 5 in Fig. 8). We obtain the same information for bended lines (illustrated in point 2 on Fig. 8). In the case of perceptual curve or junction, these informations are also similar (shown in point 8,9 and 10 on Fig. 8).

However, at the level of an edge, the absolute value of $\Sigma$ is close to 0 because the absolute values of $D$ at $\theta_{M_{1}}, \theta_{M_{2}}, \theta_{m_{1}}$ and $\theta_{m_{2}}$ are close to each other but of different signedness (see points 6 on Fig. 8).

Finally, due to the strong smoothing, sharp peaks which correspond to the directions of curves are easily extracted in the presence of noise (illustrated in point 4 and 5 in Fig. 8), that is why our method is considerately robust to noise.

\subsection{Ridges and Valleys Extraction}

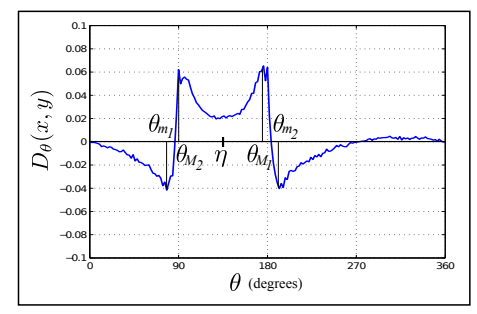

(a)

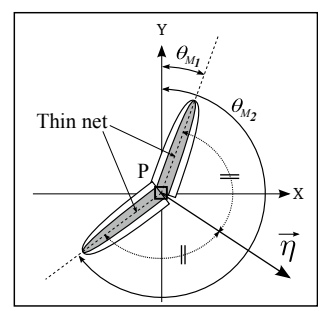

(b)
Fig. 9. $\eta$ extraction $\left(\Sigma(x, y)>\Sigma_{t h}\right)$. (a) $\eta$ computation from $\theta_{M_{1}}$ and $\theta_{M_{2}}$. (b) $\eta$ corresponds to the direction perpendicular to the curve at the level of a pixel $\mathrm{P}$.

Once $\Sigma(x, y)$ computed, we simply estimate $\eta(x, y)$ (see Fig. 9 (a) and (b)) by:

$$
\left\{\begin{array}{l}
\eta(x, y)=\left(\theta_{M_{1}}+\theta_{M_{2}}\right) / 2, \text { when } \Sigma(x, y)>\Sigma_{t h} \\
\eta(x, y)=\left(\theta_{m_{1}}+\theta_{m_{2}}\right) / 2, \text { when } \Sigma(x, y)<-\Sigma_{t h}
\end{array}\right.
$$

Thus, from $\Sigma(x, y)$ and $\eta(x, y)$ (an example in Fig. 10(b)), curves can easily be extracted computing local maxima of $\Sigma(x, y)$ in the direction $\eta(x, y)$ (for ridge detection and the minima for valley detection). Finally, we apply a hysteresis operator on the detected curves. Let us note $\tau_{L}$ the hysteresis lower threshold and $\tau_{H}$ the higher. For typical adjustment of the threshold, we adopt $\tau_{L}=\Sigma_{t h}$ and $\tau_{H}$ is tuned by the user.

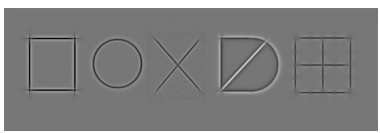

(a) $\Sigma$ image

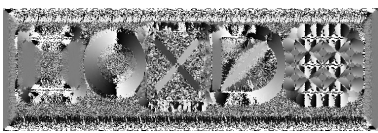

(b) $\eta$ image
Fig. 10. Example of $\Sigma$ and $\eta$ image ( $\eta$ in degrees, modulo 180) using $\Delta \theta=2$ (degrees), $\mu=10, \lambda_{1}=1, \lambda_{2}=1.5$. All these image are normalized.

\section{RESULTS}

We present results obtained both on synthetic and real images using our DRF detector. Then, we show that our method is able to extract curves and complete object contours after an edge detection in noisy real images.

\subsection{Result on Perceptual Synthetic Images}

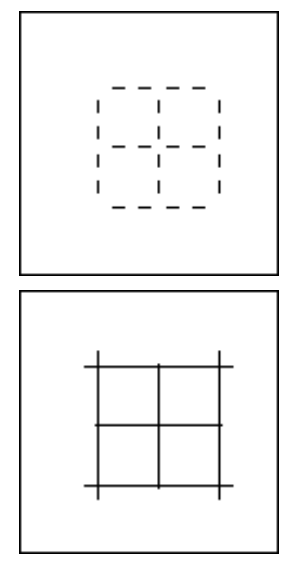

(a)

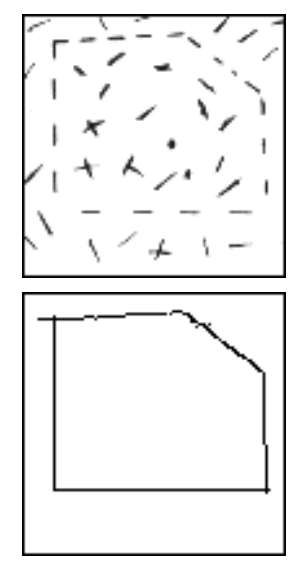

(b)

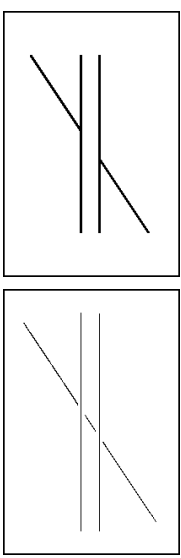

(c)
Fig. 11. Valley detection on perceptual images. Top to bottom: original image and valley detection. (a) Four perceptual squares $(118 \times 120)$. Perceptual valley detection with $\Delta \theta=2$ (degrees), $\mu=15, \lambda_{1}=1, \lambda_{2}=1.5, \tau_{L}=0.02$ and $\tau_{H}=$ 0.12 . (b) Perceptual pentagone $(100 \times 100)$. Perceptual valley detection with $\Delta \theta=2$ (degrees), $\mu=10, \lambda_{1}=1, \lambda_{2}=1.5$, $\tau_{L}=0.01$ and $\tau_{H}=0.05$. (c) Poggendorff illusion image $(210 \times 315)$. Perceptual valley detection with $\Delta \theta=2$ (degrees), $\mu=30, \lambda_{1}=1, \lambda_{2}=1.5, \tau_{L}=0.0$ and $\tau_{H}=0.04$.

In the first group of images (Fig. 11), the DRF detector is able to complete dashed lines, even for corner points. Let us 


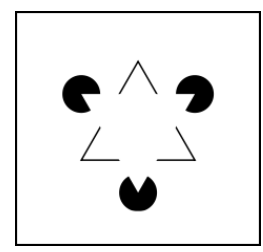

(a) Original image

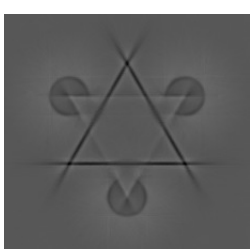

(b) $\Sigma$ image

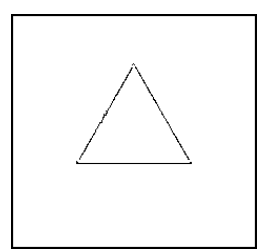

(c) Valley detection
Fig. 12. Perceptual valley detection on a Kanizsa triangle $(230 \times 220) . \Delta \theta=2$ (degrees), $\mu=20, \lambda_{1}=1, \lambda_{2}=1.5$, $\tau_{L}=0.01$ and $\tau_{H}=0.06$.

note that the noise in Fig. 11 (b) does not disturb the perceptual curve extraction.

The DRF detector has been tested on the classical Kanizsa triangle, the optical illusion in Fig. 12. Just as perceived by a human eye, the equilateral triangle can be reconstituted by the DRF, using a parameter $\mu$ large enough. Besides, a large $\mu$ does not generate any false detection.

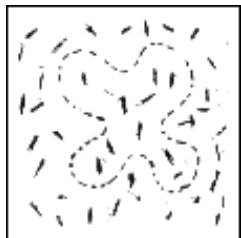

(a) Original

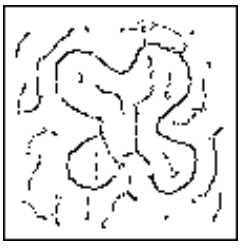

(b) Valley detection

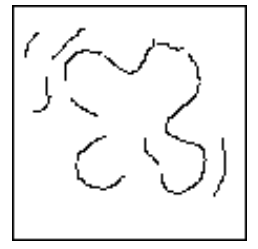

(c) Chaining
Fig. 13. Perceptual valley detection. (a) Original image (100 $\times 100$ ). (b) Perceptual valley detection result with $\Delta \theta=2$ (degrees), $\mu=5, \lambda_{1}=1.5, \lambda_{2}=2.25, \tau_{L}=0.001$ and $\tau_{H}=0.01$. (c) Chaining on (b) with a minimal size of chain equal to 12 .

In Fig. 13, the difficulty is to extract the chromosome form since small objects disturb the perception. The DRF extract correctly the form and after a chaining, we are able to reconstruct almost entirely the chromosome without presenting a lot of false perceptual curves even at the level of bended lines.

Clearly, our method fits well human perception and can be used efficiently for analysis and interpretation of images [14]. A result database is available online [15].

\subsection{Result on Real Images}

In Fig. 14(e), crests lines are not very sharp, however our detector is able to extract most of the roads and junctions. Our ridge detection superposed on the original image shows the precision and the relevancy of the DRF.

\subsection{Result on Perceptual Contours}

Edge detection can return incomplete contours and false edges, especially in the case of noisy image (illustrated in

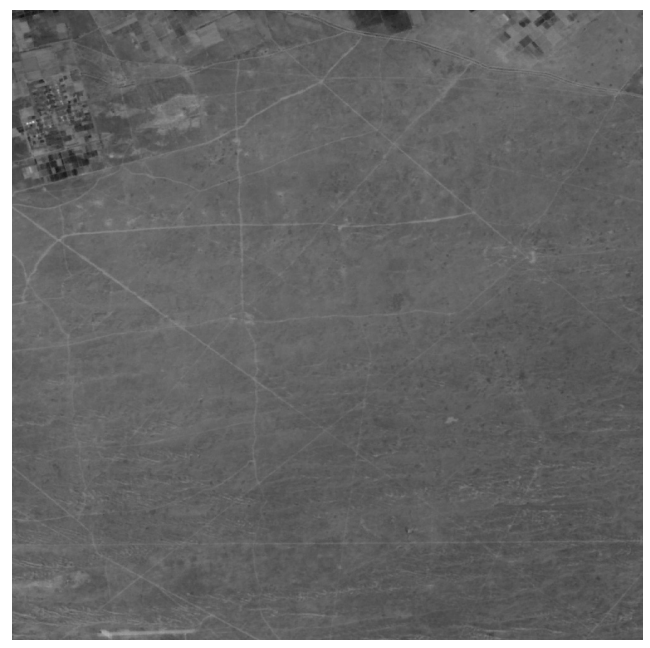

(a) Original image $1000 \times 1000$

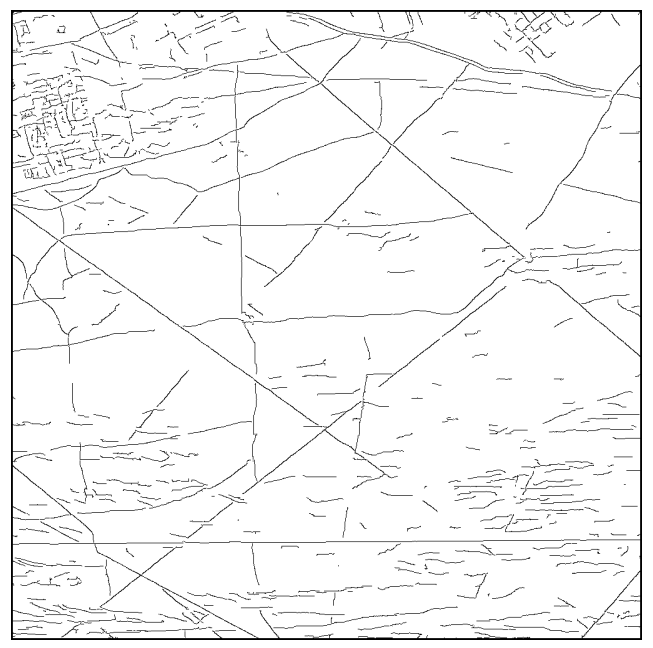

(b) Ridge detection

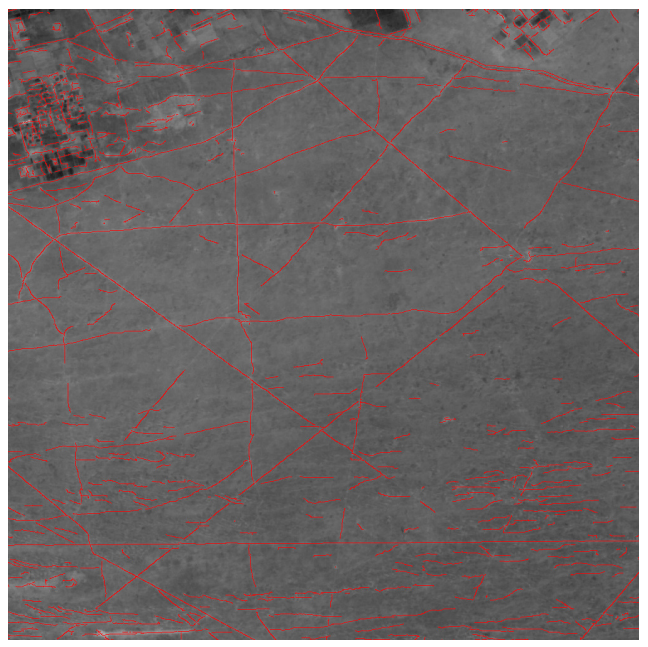

(c) Our ridge detection superposed on (a)

Fig. 14. Ridges detection on aerial images. $\Delta \theta=5$ (degrees), $\mu=10, \lambda_{1}=1, \lambda_{2}=1.5, \tau_{L}=0.001$ and $\tau_{H}=0.0025$. 
Fig. 15(b)). As the DRF detector performs in the case of perceptual curves, perceptual edges are enhanced. After two iterations, only remains objects contours [14].

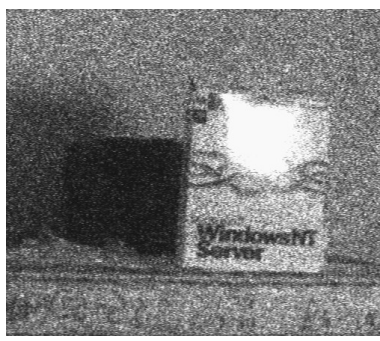

(a) Original image

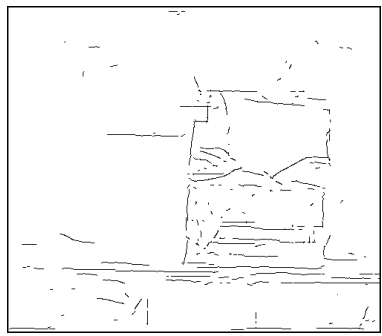

(c) Perceptual valley detection on (b)

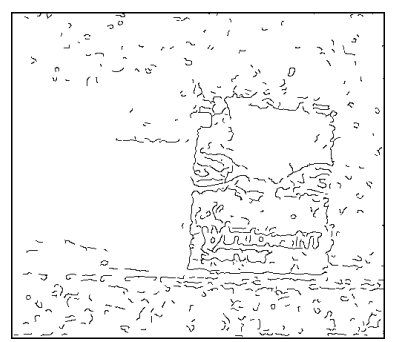

(b) Edge detection of (a)

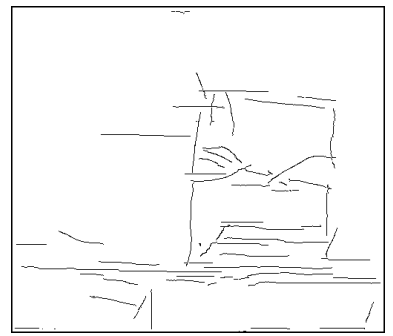

(d) Perceptual valley detection on (c)
Fig. 15. Valley detection on contours. (a) Original image $(500 \times 437)$. (b) Edge detection [11], $\sigma=2, \tau_{L}=0.03$ and $\tau_{H}=0.04$. (c) Valley detection on (b), $\Delta \theta=2$ (degrees), $\mu=20, \lambda_{1}=1, \lambda_{2}=1.5, \tau_{L}=0.02$ and $\tau_{H}=0.025$. (d) Valley detection on (c), $\Delta \theta=2$ (degrees), $\mu=20, \lambda_{1}=2$, $\lambda_{2}=3, \tau_{L}=0.01$ and $\tau_{H}=0.02$.

\section{CONCLUSION}

We have presented a new, precise and robust detection method of perceptual curves based on the difference of two rotating half smoothing linear filters and local maximization/minimization. Our method is able to detect ridges and valleys even if they are discontinuous and highly bended. Moreover, due to the use of two half rotating smoothing kernels, our approach enables to compute the two directions of a curve, which can provide important informations for example for image diffusion. Finally, the strong smoothing in the direction of the curve allows the method to be highly robust to noise. This detector has been tested successfully on various image types presenting difficult problems for classical perceptual and curve detection methods.

\section{REFERENCES}

[1] L. Alquier and P. Montesinos, "Representation of linear structures using perceptual grouping," in 1st workshop on Perceptual Organization in Computer Vision, 1998.
[2] A. Berengolts and M. Lindenbaum, "On the distribution of saliency," IEEE transactions on pattern analysis and machine intelligence, pp. 1973-1990, 2006.

[3] G. Guy and G. Medioni, "Inferring global perceptual contours from local features," International Journal of Computer Vision, vol. 20, no. 1, pp. 113-133, 1996.

[4] L. Herault and R. Horaud, "Figure-ground discrimination: A combinatorial optimization approach," IEEE Transactions on Pattern Analysis and Machine Intelligence, vol. 15, no. 9, pp. 899-914, 1993.

[5] A. A. Sha' ashua and S. S. Ullman, "Structural Saliency: The Detection of Globally Salient Structures Using Locally Connected Network," in International Conference on Computer Vision, 1988, pp. 321-327.

[6] J.M. Geusebroek, A. Smeulders, and J. Van De Weijer, "Fast anisotropic gauss filtering," Computer Vision ECCV 2002, pp. 99-112, 2002.

[7] P. Montesinos and B. Magnier, "A new perceptual edge detector in color images," in Advanced Concepts for Intelligent Vision Systems. Springer, 2010, pp. 209-220.

[8] B. Magnier, P. Montesinos, and D. Diep, "Texture Removal by Pixel Classification using a Rotating Filter," in IEEE 36th International Conference on Acoustics, Speech and Signal Processing, 2011.

[9] J. Weickert, Anisotropic diffusion in image processing, vol. 256, Citeseer, 1998.

[10] N. Armande, P. Montesinos, and O. Monga, "Thin nets extraction using a multi-scale approach," Scale-Space Theory in Computer Vision, pp. 361-364, 1997.

[11] J. Canny, "A computational approach to edge detection," Readings in computer vision: issues, problems, principles, and paradigms, vol. 184, 1987.

[12] T. Lindeberg, "Edge detection and ridge detection with automatic scale selection," International Journal of Computer Vision, vol. 30, no. 2, pp. 117-154, 1998.

[13] R. Deriche, "Recursively Implementing the Gaussian and Its Derivatives," in Proc. Second International Conference On Image Processing, Singapore, Sept. 7-11 1992, pp. 263-267.

[14] D.G. Lowe, Perceptual organization and visual recognition, Ph.D. thesis, Stanford University, 1984.

[15] B. Magnier, D. Diep, and P. Montesinos, "DRF results," http://www.lgi2p.ema.fr/ magnier/ Demos/DRFresults.html. 